\title{
Comparison of the clinical and microbiological effects of antibiotic therapy in periodontal pockets following laser treatment: An in vivo study
}

\author{
Kinga Grzech-Leśniak ${ }^{1, A-F}$, Jacek Matys ${ }^{1,2, C, D}$, Marzena Dominiak1,E,F \\ ${ }^{1}$ Department of Dental Surgery, Wroclaw Medical University, Poland \\ 2 Private Dental Practice, Wschowa, Poland \\ A - research concept and design; B - collection and/or assembly of data; $C$ - data analysis and interpretation; \\ $\mathrm{D}$ - writing the article; $\mathrm{E}$ - critical revision of the article; $\mathrm{F}$ - final approval of the article
}

\section{Address for correspondence \\ Jacek Matys}

E-mail: jacek.matys@wp.pl

Funding sources

None declared

Conflict of interest

None declared

Received on December 29, 2016

Reviewed on February 5, 2017

Accepted on April 12, 2017

DOI

10.17219/acem/70413

\section{Copyright}

Copyright by Author(s)

This is an article distributed under the terms of the

Creative Commons Attribution Non-Commercial License

(http://creativecommons.org/licenses/by-nc-nd/4.0/)

\begin{abstract}
Background. Laser technology in periodontal therapy could help in reducing total bacterial count.

Objectives. The aim of this study was to evaluate the effects of pocket debridement using an erbium-doped yttrium aluminium garnet laser (Er:YAG laser - ERL), scaling and root planing (SRP) with photodynamic therapy (PDT), or SRP alone. Teeth vitality and soft tissue carbonization were also assessed.

Material and methods. This study included 1,169 single-rooted teeth from 84 patients divided into 3 groups $(n=28)$. The $\mathrm{G} 1$ group had ERL with $40 \mathrm{~mJ}$ of energy, a frequency of $40 \mathrm{~Hz}$ and a fluence of $63.66 \mathrm{~J} / \mathrm{cm}^{2}$. The $\mathrm{G} 2$ group had SRP + PDT (635 nm diode laser, $12 \mathrm{I}$ of energy and irradiation time of $30 \mathrm{~s}$ ) and a Toluidine Blue photosensitizer (PS) (application time of $60 \mathrm{~s}$ ). The G3 group was administered SRP alone. In the 42 subjects (G1: $n=11, G 2: n=14$ and G3: $n=17$ ) with high amounts of Aggregatibacter actinomycetemcomitans (Aa), Porphyromonas gingivalis (Pg), Treponema denticola (Td) and Tannerella forsythia (Tf), additional 1-week antibiotic treatments with clindamycin or amoxicillin + clavulanic acid - in doses of $600 \mathrm{mg} / \mathrm{day}$ or $1000 \mathrm{mg} / \mathrm{day}$, respectively - were prescribed 3 months after the therapy. Microbiological and clinical analyses of the probing depth (PD), recession (RC), plaque index (PI), bleeding on probing (BOP), and attachment loss (AT) were performed at baseline and at the follow-up of 3 months, 3 months and 1 week, and 6 months.
\end{abstract}

Results. Plaque index decreased in $\mathrm{G} 1$ after 3 months, 3 months and 1 week, and 6 months $(\mathrm{p}<0.05)$ and was lower in G1 vs G2 after 3 months $(p<0.05)$. The reduction in BOP in $G 1$ after 3 months and 1 week was higher in comparison with $G 2$ or G3 $(p<0.02)$. Probing depth decreased in all groups $(p<0.05)$. We found a reduction in the percentage of sites with some bacteria after 3 months - Prevotella intermedia (Pi) (G1 and G2), Capnocytophaga gingivalis (Gg) and Eubacterium nucleatum (En) (G3), and after 3 months and 1 week with En, Td, Tf (G1, G2 and G3), Pi (G1 and G2), Aa, Peptostreptococcus micros (Pm), and Cg (G3), and with $P i$ (G1 and $G 2)$, If (G2), $P g$, En (G2 and $G 3)$, and $P m(G 3)$ after 6 months $(p<0.05)$. We observed no signs of carbonization or teeth injury.

Conclusions. Scaling and root planing + PDT and ERL may be an alternative therapy for chronic periodontitis.

Key words: chronic periodontitis, photodynamic therapy, antibacterial therapy, scaling and root planning, Er:YAG laser 


\section{Introduction}

Recently, more and more bacterial strains have become resistant to antibiotics. ${ }^{1}$ The excessive use of antibiotics and the development of several new ones by pharmaceutical companies are 2 reasons why some researchers describe the first decade of the $21^{\text {st }}$ century as the antibiotic resistance crisis. ${ }^{2-4}$ Anaerobic bacterial growth in the periodontal pocket leads to periodontal diseases, which are becoming more and more common in Poland; they also increase the risk of tooth loss or other medical complications, such as acute myocardial infarction or stroke. ${ }^{5-7}$

Clinical and microbiological analyses using traditional culture methods and the polymerase chain reaction (PCR) test provide general data about the severity of periodontitis, which are useful in designing treatment strategies. ${ }^{8}$ Unfortunately, the basic PCR technique does not provide a quantitative assessment of the identified bacteria. ${ }^{9}$ Due to its ability to generate both qualitative and quantitative results, the newer and more costly real-time polymerase chain reaction (rtPCR) analysis has provided scientific advancement. ${ }^{9}$

The gold standard in periodontal treatment is the proper eradication of pathological bacterial strains inside the infected pockets by means of mechanical debridement. ${ }^{10,11}$ However, root architecture and its particular anatomical variations are an obstruction in establishing effective protocols for debridement in non-surgical periodontal therapy, which uses traditional tools, such as curettes or ultrasonic scalers. ${ }^{12}$ Therefore, different devices (e.g., lasers) have been used in non-surgical periodontal treatment as alternative or adjunct methods to mechanical scaling and root planing (SRP). Many clinical studies have confirmed the effectiveness of the $2940 \mathrm{~nm}$ erbium-doped yttrium aluminium garnet laser (Er:YAG laser - ERL) in periodontal therapy, with or without additional SRP. ${ }^{13-15}$ The combination of low-power lasers and different photosensitizers (PSs), known as antimicrobial photodynamic therapy (aPDT), enhanced the elimination of periopathogenic bacteria and the reduction of bone loss. ${ }^{16-18}$ Furthermore, the ability of lasers to decontaminate the root surface with minimal (ERL) or no damage (PDT) could be of great value in periodontal therapy, because mechanical instruments may lead to excessive removal of root material. ${ }^{19}$ However, in their study, Zach and Cohen reported that an intrapulpal temperature increase of $5.5^{\circ} \mathrm{C}$ caused pulpitis or pulp necrosis in $15 \%$ of the irradiated teeth. ${ }^{20}$ Thus, particular attention should be paid to preventing thermal injury of periodontal tissues when high-powered lasers are applied during periodontal procedures.

\section{Objectives}

The aim of this randomized, controlled clinical trial was to compare the clinical and microbiological effects of antibiotic therapy following pocket debridement, using ERL and SRP + PDT as compared to SRP alone. Additionally, the objective was to assess the vitality of the teeth after periodontal procedures and the occurrence of soft tissue carbonization.

\section{Material and methods}

The trial was designed as a randomized and controlled test lasting 6 months. The study protocol was reviewed and approved by the Bioethics Commission of the Regional Chamber of Physicians and Dentists in Kraków (permission No.: 74/KBL/OIL/2014). Informed consent was obtained from all participating subjects.

\section{Subjects}

The study included 1,169 periodontally-involved single-rooted teeth in 84 patients (38 women and 46 men, aged $48.6 \pm 9.4$ years). All patients were diagnosed with generalized chronic periodontitis according to the classification of periodontal diseases from the American Academy of Periodontology (AAP) classification from 1999. ${ }^{21}$ The groups were treated at the private clinic PerioCare in Kraków, Poland. Selected patients in the study: 1 . had all single-rooted teeth with a probing depth (PD) of $\geq 5 \mathrm{~mm}$ and no signs of apical pathology; 2 . had no systemic diseases; 3 . were not using anti-inflammatory drugs; 4 . had not used antibiotics for the previous 24 months; 5 . were non-smokers; 6 . had not undergone periodontal treatment before the trial; 7 . had undergone hygienist treatment before the clinical trial.

\section{Treatment procedures}

A total of 84 patients were randomly assigned by a computer to the 3 study groups according to the treatment procedure: G1 - ERL ( $n=28) ; \mathrm{G} 2$ - SRP + PDT ( $=28)$; and $\mathrm{G} 3$ - SRP alone $(\mathrm{n}=28)$. For the 592 periodontally-involved single-rooted teeth in 42 subjects (G1: $\mathrm{n}=11, \mathrm{G} 2: \mathrm{n}=14$ and G3: $\mathrm{n}=17$ ) with high amounts of Aggregatibacter actinomycetemcomitans (Aa), Porphyromonas gingivalis (Pg), Treponema denticola (Td), and Tannerella forsythia (Tf), additional antibiotic treatments were prescribed after the therapy according to the antibiogram: clindamycin (Clindamycin-MIP ${ }^{\circledR}$; MIP Pharma, Innsbruck, Austria) or amoxicillin + clavulanic acid (Amoksiklav; Sandoz, Warszawa, Poland), in dosages of $600 \mathrm{mg} /$ day or $1000 \mathrm{mg} /$ day for 1 week, respectively.

\section{Clinical treatment}

In each patient, non-surgical periodontal pocket debridement was performed by a specialist in the area of periodontal treatment, using different therapeutic methods.

Group 1 received periodontal debridement with the Er:YAG laser (LightWalker; Fotona, Ljubljana, Slovenia) 
with the following fixed operation parameters: pulse mode - medium-short pulse (MSP), energy - $40 \mathrm{~mJ}$, frequency - $40 \mathrm{~Hz}$, fluence per point $-63.66 \mathrm{~J} / \mathrm{cm}^{2}$, perio tip (Varian) $-400 \mu \mathrm{m}$, and water spray $-100 \%$. Laser therapy was performed by inserting the fiber into the periodontal pocket almost parallelly to the tooth and moving in a mesial to distal direction continuously on the buccal and lingual surface of the tooth, 10 to $30 \mathrm{~s}$ per tooth.

Group 2 received periodontal debridement with a combination of SRP and Photoactive Disinfection with a Diode laser (Smart M; Lasotronix, Piaseczno, Poland) with the following fixed operation parameters: continuous wave mode and $200 \mathrm{~mW}$ of energy. Photosensitizer (Toluidine Blue 0.1\%) was applied into the pocket and left for $60 \mathrm{~s}$; then, the tip of the Diode laser (PACT ${ }^{\circledR}$ Light Guides Universal; Cumdente GmbH, Tübingen, Germany) was inserted into the pocket subgingivally and the PS was irradiated for $30 \mathrm{~s}$.

Group 3 (control) received SRP alone - periodontal debridement, using hand curettes (Double Gracey XP; American Eagle Instruments, Inc., Missoula, USA).

\section{Clinical parameters}

Clinical data was collected before treatment (baseline) and at follow-up examinations after 3 months, 3 months and 1 week, and 6 months. All recordings in each patient were taken with a fully automated periodontal probe (paon; Orangedental GmbH \& Co.KG, Biberach an der Riss, Germany), which transfers the data onto a computer screen.

The following clinical parameters were evaluated:

- plaque index (PI): evaluation of the presence of plaque at the cervical margin of the tooth, using a periodontal probe, expressed as percentage;

- pocket depth (PD): the distance from the gingival margin to the bottom of the pocket, measured at 6 sites of the single-rooted teeth (mesiobuccal/midbuccal/distobuccal/mesiopalatal/midpalatal/distopalatal) with a constant probing force of $0.2 \mathrm{~g}$ of pressure;

- bleeding on probing (BOP): determined by the presence $(+)$ or absence $(-)$ of bleeding for $30 \mathrm{~s}$ after the first probe was inserted into the pocket, expressed as percentage;

- recession ( $\mathrm{RC})$ : distance from the cemento-enamel junction (CEJ) to the gingival margin (and from the crown margin to the gingival margin);

- attachment loss (AT): the distance between the CEJ and the base of the sulcus; it includes both PD and RC measurements.

\section{Microbiological analysis}

The microbiological examination of every patient consisted of a biological-molecular test (PetPlus; MIP Pharma GmbH, Blieskastel-Niederwürzbach, Germany) which involves the detection of 9 periodontal pathogens, including red, orange, green, and yellow complexes (e.g., $\mathrm{Aa}, \mathrm{Pg}, \mathrm{Td}$, Tf, Prevotella intermedia - Pi, Peptostreptococcus micros - Pm, Fusobacterium nucleatum - Fn, Eubacterium nucleatum - En, and Capnocytophaga gingivalis $-\mathrm{Cg}$ ). The test was performed by means of the rtPCR method. The samples were taken according to the manual with enclosed sterile paper swabs, so the uniformity of the sampling was ensured. The paper swabs were inserted into the gingival pockets for $30 \mathrm{~s}$.

Microbiological analysis by means of rtPCR was conducted: 1 . at baseline; 2.3 months after the treatment in all 3 groups; 3 . 1 week after introducing the additional antibiotic therapy (Clindamycin-MIP or Amoksiklav, duration: 1 week); 4 . 3 months after the antibiotic therapy (6 months since baseline).

\section{Tissue injury test}

The visual signs of soft tissue carbonization and the reaction of the teeth (+/-) to ethyl chloride after the treatment were evaluated.

\section{Statistical analysis}

All data was subjected to statistical analyses with STATISTICA 12 software (StatSoft, Kraków, Poland). The differences in the clinical parameters (PI, PD, BOP, RC, and $\mathrm{AT}$ ) in the time periods and between the groups were analyzed according to the 2-way repeated analysis of variance (ANOVA) test. The Cochran's Q test and the $\chi^{2} \mathrm{McNemar}$ exact test were used to assess bacterial prevalence. Values of $\mathrm{p} \leq 0.05$ were considered to be significant.

\section{Results}

A total of 1,169 periodontally-involved single-rooted teeth in 84 patients were included in this study.

\section{Plaque index}

In the comparison between the baseline and 3 months (8.4\% to $4.6 \%), 3$ months and 1 week (8.4\% to $5.8 \%)$, and 6 months $(8.4 \%$ to $4.0 \%)$ after the treatments, there was a significant reduction in PI $(\mathrm{p}<0.05)$ in the G1 group (Fig. 1).

The difference among the groups revealed a significantly lower PI value after 3 months in the G1 group as compared to the G2 group ( $\mathrm{p}<0.01$ ) (Fig. 2).

\section{Bleeding on probing}

A significant reduction in BOP after an additional antibiotic treatment at 3 months and 1 week, and 6 months, in both the G1 and G2 groups, was observed compared with the G3 group (p < 0.01) (Fig. 3). 


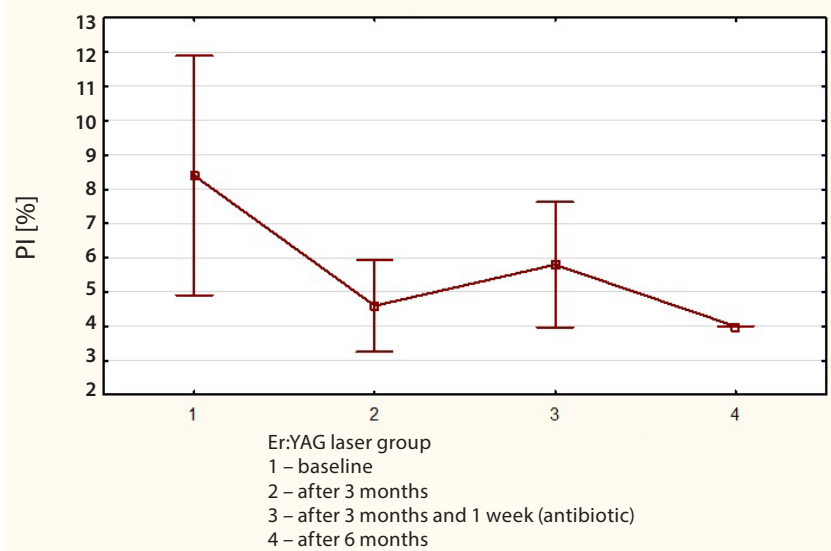

Fig. 1. The mean value of Pl after periodontal pocket debridement with Er:YAG laser at 4 time points

$\mathrm{Pl}$ - plaque index.

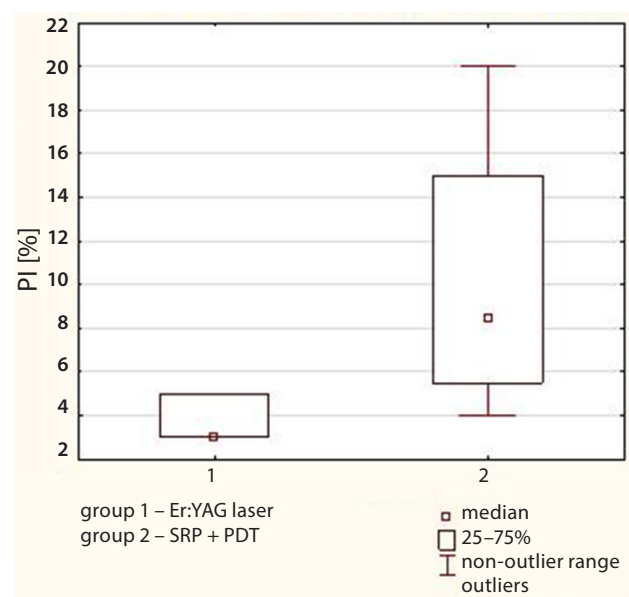

Fig. 2. The results of PI 3 months after periodontal pocket debridement with different treatment methods

PI - plaque index; SRP - scaling and root planing; PDT - photodynamic therapy.

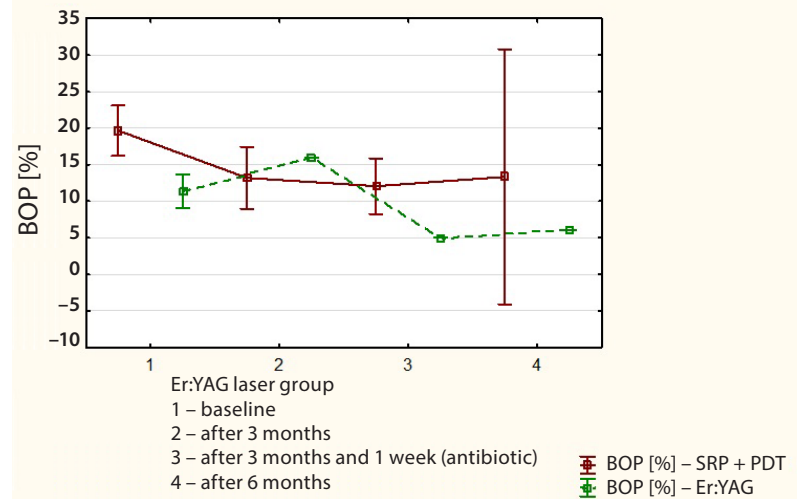

Fig. 3. The mean value of $B O P$ after periodontal pocket debridement with Er:YAG laser or SRP + PDT at 4 time points

BOP - bleeding on probing; SRP - scaling and root planing; PDT - photodynamic therapy.

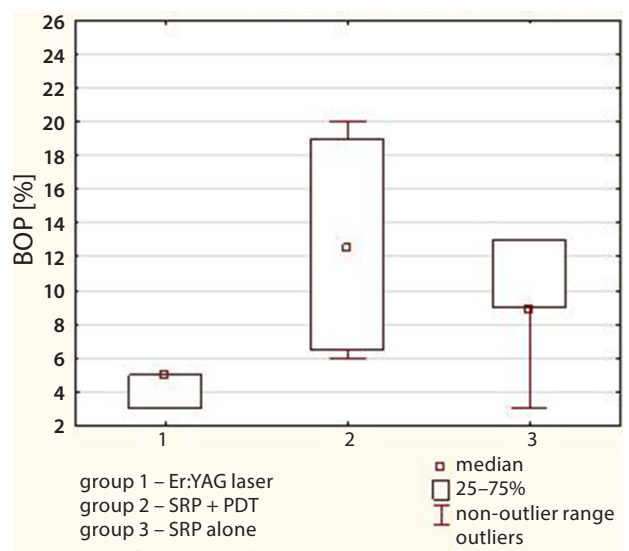

Fig. 4. The results of BOP after periodontal pocket debridement with Er:YAG laser, SRP + PDT or SRP alone 1 week after additional antibiotics delivery

BOP - bleeding on probing; SRP - scaling and root planing;

PDT - photodynamic therapy.

Table 1. The mean reduction in PD [mm] for all 3 patient groups after 3 months, 3 months and 1 week, and 6 months

\begin{tabular}{|l|c|c|c|c|}
\hline \multicolumn{1}{|c|}{ Study groups } & Baseline & 3 months & 3 months 1 week & 6 months \\
\hline G1 & $6.12 \pm 1.95$ & $2.88 \pm 0.75$ & $2.72 \pm 0.65$ & $2.95 \pm 0.67$ \\
\hline G2 & $6.7 \pm 1.73$ & $2.76 \pm 0.61$ & $2.5 \pm 0.53$ & $2.6 \pm 0.49$ \\
\hline G3 & $6.03 \pm 1.41$ & $2.55 \pm 0.58$ & $2.66 \pm 0.53$ & $2.89 \pm 0.58$ \\
\hline p-value & NS & NS & NS & NS \\
\hline
\end{tabular}

G1 - group with ERL; G2 - group with SRP + PDT; G3 - group with SRP alone; PD - pocket depth; ERL - Er:YAG laser; SRP - scaling and root planing; PDT - photodynamic therapy; NS - not significant. Data is presented as mean \pm standard deviation (SD).

The additional delivery of antibiotics resulted in a decrease in the BOP level in the G1 group as compared with the G2 and G3 groups ( $<$ <.02) (Fig. 4).

\section{Pocket depth}

There was a significant difference in the reduction of PD in all 3 patient groups after 3 months, 3 months and 1 week, and 6 months as compared with the baseline $(\mathrm{p}<0.05)$ (Table 1).

\section{Recession and attachment loss}

The comparison of RC and AT showed insignificant results among all groups and between all study periods $(\mathrm{p}>0.05)$ (Tables 2, 3).

\section{Microbiological results}

After 3 months, we observed a reduction of $P i$ in the laser-treated groups G1 and G2, of $C g$ and $E n$ in the G3 
Table 2. The mean RC size [mm] for all 3 patient groups after 3 months, 3 months and 1 week, and 6 months

\begin{tabular}{|l|c|c|c|c|}
\hline \multicolumn{1}{|c|}{ Study groups } & Baseline & 3 months & 3 months 1 week & 6 months \\
\hline G1 & $1.1 \pm 0.75$ & $1.19 \pm 0.78$ & $1.22 \pm 0.73$ & $1.11 \pm 0.72$ \\
\hline G2 & $0.8 \pm 0.62$ & $0.96 \pm 0.52$ & $0.99 \pm 0.38$ & $0.97 \pm 0.49$ \\
\hline G3 & $1.13 \pm 0.75$ & $1.19 \pm 0.79$ & $1.1 \pm 0.8$ & NS \\
\hline p-value & NS & NS & NS & NS \\
\hline
\end{tabular}

G1 - group with ERL; G2 - group with SRP + PDT; G3 - group with SRP alone; RC - recession; ERL - Er:YAG laser; SRP - scaling and root planing; PDT - photodynamic therapy; NS - not significant. Data is presented as mean \pm standard deviation (SD).

Table 3. The mean AT [mm] for all 3 patient groups after 3 months, 3 months and 1 week, and 6 months

\begin{tabular}{|l|c|c|c|c|}
\hline \multicolumn{1}{|c|}{ Study groups } & Baseline & 3 months & 3 months 1 week & 6 months \\
\hline G1 & $3.22 \pm 0.77$ & $3.03 \pm 0.92$ & $2.93 \pm 1.03$ & $4.02 \pm 1.73$ \\
\hline G2 & $3.25 \pm 1.09$ & $3.13 \pm 0.98$ & $3.09 \pm 0.67$ & $3.26 \pm 0.91$ \\
\hline G3 & $3.15 \pm 0.77$ & $3.04 \pm 0.97$ & $2.76 \pm 1.29$ & $2.99 \pm 1.32$ \\
\hline p-value & NS & NS & NS & NS \\
\hline
\end{tabular}

G1 - group with ERL; G2 - group with SRP + PDT; G3 - group with SRP alone; AT - attachment loss; ERL - Er:YAG laser; SRP - scaling and root planing; PDT - photodynamic therapy; NS - not significant. Data is presented as mean \pm standard deviation (SD).

group, and a significant increase in $A a$ (G2 and G3), $P i$ (G3), and $P g$ (in all 3 study groups) (p $<0.05$ ).

After 3 months and 1 week, we noted a reduction of $T d$, $T f$ and $E n$ in all 3 groups, of $P i$ in the G1 and G2 groups, $A a, P m, C g$ in the G3 group, and a significant increase in $A a$ in the $\mathrm{G} 2$ group ( $<0.05$ ).

After 6 months, a reduction in the percentage of sites with bacterial prevalence of $P i$ (G1 and G2), Tf (G2), $P g$, En (G2 and G3), and Pm (G3) was reported as compared to the baseline $(\mathrm{p}<0.05)$ (Fig. 5).

Additionally, we observed no signs of carbonization during periodontal pocket debridement with the Er:YAG laser with the fixed operation parameters used in this study (energy $40 \mathrm{~mJ}$, frequency $-40 \mathrm{~Hz}$, fluence per point $\left.-63.66 \mathrm{~J} / \mathrm{cm}^{2}\right)$. The pulp vitality test in all the teeth indicated a positive reaction to ethyl chloride following treatment.

\section{Discussion}

A review of the literature to date provides no record of a comparison of in vivo studies which evaluated the clinical and microbiological effects of antibiotic therapy following pocket debridement, using ERL and SRP + PDT in the same study. Furthermore, the impact of additional antibiotic therapy on bacterial counts in periodontal pockets, assessed by means of the rtPCR test has not been analyzed in relation to previous laser applications. The main objective of this study was to determine the effects of different periodontal pocket debridement methods (ERL and SRP + PDT) on clinical parameters, such as PI, PD, BOP, AT, RC, and bacterial count in the rtPCR test (PetPlus test).

The results of our study show a significant reduction in PD of $6.12 \mathrm{~mm}$ to $2.95 \mathrm{~mm}, 6.7 \mathrm{~mm}$ to $2.6 \mathrm{~mm}$ and $6.03 \mathrm{~mm}$ to $2.89 \mathrm{~mm}$ in the groups G1, G2 and G3 after 6 months, respectively. Our findings were consistent with the results presented by other authors for different laser wavelengths, e.g., $2940 \mathrm{~nm},{ }^{14} 2780 \mathrm{~nm},{ }^{22} 940 \mathrm{~nm},{ }^{22}$ and for aPDT ${ }^{23,24}$ and SRP alone. ${ }^{14}$ All the methods of treating chronic periodontitis discussed in the present study were efficient and resulted in a reduction of PD; however, there were no significant differences identified between the treatment groups, which was confirmed in another study. ${ }^{25}$

The second tested outcome parameter was the BOP score. Several authors indicated significant improvements in BOP after erbium laser irradiation ${ }^{13-15,22}$ or PDT. ${ }^{23}$ We found an insignificant increase in BOP after the application of the Er:YAG laser. Ciurescu et al. also reported a lower reduction in the BOP score, but only when the Er:YAG laser and SRP were compared at 2 months' follow-up (32\% vs 38\%). ${ }^{22}$

Surprisingly, we found no significant differences among all treatment methods with respect to AT and RC size at each time point. Rotundo et al. in their study also showed that there was no significant difference between ERL + SRP and SRP alone in the clinical attachment level in a group of 27 patients who underwent non-surgical periodontal therapy. These results were confirmed by Tomasi et al., comparing the Er:YAG laser and the ultrasonic scaler at a 4-month follow-up examination. ${ }^{25,26}$ On the other hand, Moreira et al. demonstrated different results, as they found clinical attachment gain to be significantly higher after the application of 4 sessions of aPDT than in the control group (SRP). ${ }^{27}$

Particular attention in our study was paid to the influence of additional antibiotic therapy on the pathogenic bacteria which colonize the periodontal pockets. Regardless of the reduction in PD and the BOP index after Er:YAG laser debridement and the combination of SRP + PDT therapy, bacteria in the periodontal pocket were not completely eliminated. While laser application helped reduce bacterial counts without any side effects for the 

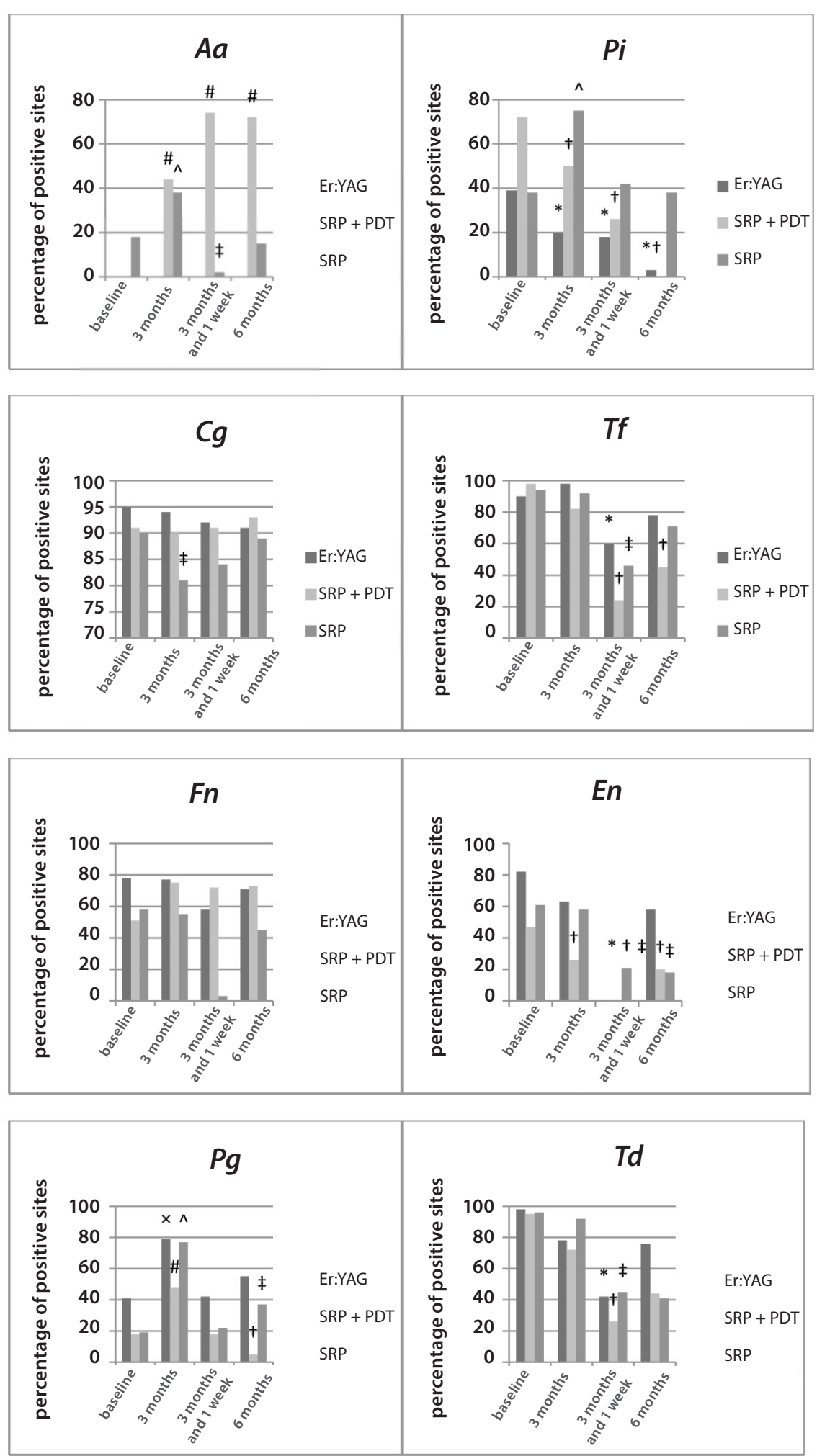

Fig. 5. The microbiological examination of different periodontal pathogens, using the rtPCR test at baseline, 3 months, 3 months and 1 week, and 6 months

rtPCR - real-time polymerase chain reaction; SRP - scaling and root planing; PDT - photodynamic therapy; Aa-Aggregatibacter actinomycetemcomitans; $\mathrm{Pi}$ - Prevotella intermedia; Cg-Capnocytophaga gingivalis; Tf-Tannerella forsythia; Fn - Fusobacterium nucleatum; En - Eubacterium nucleatum; Pg-Porphyromonas gingivalis: $T d$ - Treponema denticola. Significant reduction in the percentage of sites positive to microbial testing in comparison with the baseline for: * Er:YAG laser (G1); † SRP + PDT (G2); ₹ SRP (G3); $\times$ Er:YAG laser (G1); \# SRP + PDT (G2); ^ SRP (G3). patient, the goal was to eradicate any bacterial strains from the periodontal pocket. Our study design involved only 1 application of the laser, which resulted in incomplete elimination of bacteria. The remaining bacteria may be eradicated by additional antibiotic treatment, as suggested by the American Academy of Periodontology and the European Federation of Periodontology. ${ }^{28}$ However, to date there have been no established protocols or guidelines on the optimal additional antibiotic therapy recommended in such cases. It remains a challenge for the dental community to design protocols that will reduce the use of systemic antibiotics in favor of local ones. Generally speaking, we should aim at a minimally invasive treatment, and laser therapy is exactly that.

We conducted a microbiological analysis of the bacterial strains in the periodontal pockets after the use of the 
erbium laser and PDT. The success of these treatments can be attributed to the efficient removal of the subgingival biofilm and the eradication of bacteria from the root surface. ${ }^{12}$ Similarly to the findings of Lopes et al., our trial showed a significant reduction in the percentage of sites with $P i$ and $P g$ after erbium laser irradiation. ${ }^{14}$ However, there is a fundamental difference in the response to PDT between Gram-positive and Gram-negative bacteria. Gram-negative bacteria consist of an inner cytoplasmic membrane and an outer membrane, while Gram-positive bacteria are composed of only a cytoplasmic membrane surrounded by a relatively porous cell wall composed of lipoteichoic acid and peptidoglycan. The differences in their composition result in different susceptibility and permeability to chemicals. ${ }^{24}$ It was found that neutral, anionic and cationic PS molecules efficiently kill Grampositive bacteria, whereas only cationic PS molecules are able to kill Gram-negative strands. ${ }^{24}$ However, some modified non-cationic PS, such as chlorin (e6), have the ability of anchoring to the negatively charged lipopolysaccharide molecules. Unfortunately, studies using this dye in aPDT in periodontal research are scarce. Hence, additional alternatives to antibiotic therapy with different non-cationic PS during periodontal therapy should be researched.

We also evaluated the influence of additional antibiotic treatment on bacterial counts. The levels of bacteria strains such as $A a, P g, T d$, and $T f$ remained high after periodontal treatment using the tested therapeutic methods (ERL, SRP + PDT and SRP alone). We confirmed that antibiotics caused a decrease in the number of $P g$, $T d$ and $T f$ bacteria. Unfortunately, the overuse of antibiotics contributes to the development of bacterial resistance, thus careful patient selection, proper antibiotic treatment protocols and guidelines, the use of antibiograms, and - most of all - new nonantibiotic treatment methods require rigorous research and development. ${ }^{29,30}$

In comprehensive periodontal treatment, lasers offer a non-invasive alternative or complementary therapy method. The use of lasers in repeated therapy could help decrease the number of periopathogenic bacteria and keep their counts at a stable, low level. Hence, we are of the opinion that laser therapy constitutes a promising alternative and calls for more research to be conducted for the repeated therapy of periodontal diseases by means of the Er:YAG laser and PDT.

\section{Conclusions}

The current study failed to confirm significant differences in many compared clinical outcome parameters for non-surgical periodontal treatments with Er:YAG laser debridement, SRP + PDT therapy and the conventional SRP procedure alone. It did, however, show that additional systemic antibiotic administration significantly decreased BOP in the G1 group. The microbiological analysis in the present study revealed somewhat better results after nonsurgical periodontal treatment with the Er:YAG laser than after a combination of SRP with PDT, which had little clinical effect on the eradication of red complex bacteria. Additional antibiotic therapy with clindamycin or amoxicillin+clavulanic acid had a positive effect on the reduction of $T d$ and $T f$ after periodontal pocket debridement with ERL, SRP + PDT and SRP alone.

The use of combined antibiotic therapy and Er:YAG laser treatment may be a valuable therapeutic option in the treatment of periodontal diseases and it demands further research.

\section{References}

1. Wright GD. Something new: Revisiting natural products in antibiotic drug discovery. Can J Microbiol. 2014;60(3):147-154.

2. Ventola CL. The antibiotic resistance crisis: Part 1: Causes and threats. P\&T. 2015;40(4):277-283.

3. Piddock LJ. The crisis of no new antibiotics - what is the way forward? Lancet Infect Dis. 2012;12(3):249-253.

4. Michael CA, Dominey-Howes $D$, Labbate $M$. The antibiotic resistance crisis: Causes, consequences, and management. Front Public Health. 2014;2:145.

5. Górska R, Pietruska M, Dembowska E, Wysokińska-Miszczuk J, Włosowicz M, Konopka T. Prevalence of periodontal diseases in 35-44 year-olds in the large urban agglomerations. Dent Med Probl. 2012;49:19-27.

6. Górski B, Nargiełło E, Grabowska E, Opolski G, Górska R. The association between dental status and risk of acute myocardial infarction among poles: Case-control study. Adv Clin Exp Med. 2016;25(5): 861-870.

7. Slowik J, Wnuk MA, Grzech K, et al. Periodontitis affects neurological deficit in acute stroke. J Neurol Sci. 2010;15:297(1):82-84.

8. Yoshida A, Ansai T. Microbiological diagnosis for periodontal diseases. In: Manakil J, ed. Periodontal Diseases - A Clinician's Guide. Rijeka, Croatia: InTech; 2012:55.

9. Valones MA, GuimarãesRL, BrandãoLA, Souza PR, Carvalho AD, CrovelaS. Principles and applications of polymerase chain reaction in medical diagnostic fields: A review. Braz J Microbiol. 2009;40(1):1-11.

10. Sanz I, Alonso B, Carasol M, Herrera D, Sanz M. Nonsurgical treatment of periodontitis. J Evid Base Dent Pract. 2012;12(3):76-86.

11. Graetz C, Plaumann A, Wittich $R$, et al. Removal of simulated biofilm: An evaluation of the effect on root surfaces roughness after scaling. Clin Oral Invest. 2016;27:1-8.

12. Graetz C, Plaumann A, Bielfeldt J, Tillner A, Sälzer S, Dörfer CE. Efficacy versus health risks: An in vitro evaluation of power-driven scalers. J Indian Soc Periodontol. 2015;19(1):18.

13. Gutknecht N, Van Betteray C, Ozturan S, Vanweersch L, Franzen R. Laser supported reduction of specific microorganisms in the periodontal pocket with the aid of an Er,Cr:YSGG laser: A pilot study. Sci World J. 2015;2015:450258. doi:10.1155/2015/450258

14. Lopes BM, Theodoro LH, Melo RF, Thompson GM, Marcantonio RA. Clinical and microbiologic follow-up evaluations after non-surgical periodontal treatment with erbium: YAG laser and scaling and root planing. J Periodontol. 2010;81(5):682-691.

15. Sgolastra F, Petrucci A, Gatto R, Monaco A. Efficacy of Er:YAG laser in the treatment of chronic periodontitis: Systematic review and meta-analysis. Laser Med Sci. 2012;27(3):661-673.

16. Talebi M, Taliee R, Mojahedi M, Meymandi M, Torshabi M. Microbiological efficacy of photodynamic therapy as an adjunct to non-surgical periodontal treatment: A clinical trial. J Lasers Med Sci. 2016;(2): 126-130.

17. Nielsen HK, Garcia J, Væth M, Schlafer S. Comparison of riboflavin and toluidine blue $\mathrm{O}$ as photosensitizers for photoactivated disinfection on endodontic and periodontal pathogens in vitro. PloSONE. 2015;10(10):e0140720. 
18. Prates RA, Yamada AM, Suzuki LC, et al. Histomorphometric and microbiological assessment of photodynamic therapy as an adjuvant treatment for periodontitis: A short-term evaluation of inflammatory periodontal conditions and bacterial reduction in a rat model. Photomed Laser Surg. 2011;29:835-844.

19. de Oliveira GJ, Cominotte MA, BeraldoTP, Sampaio JE, Marcantonio RA. A microscopic analysis of the effects of root surface scaling with different power parameters of Er, Cr: YSGG laser. Microsc Res Techniq. 2015;78(6):529-535.

20. Zach L, Cohen G. Pulp response to externally applied heat. Oral Surg Oral Med Oral Pathol. 1965;19:515-530.

21. Armitage GC. Development of a classification system for periodontal diseases and conditions. Ann Periodontol. 1999:4:1-6.

22. Ciurescu C, Teslaru S, Zetu L, Ciurescu D. Laser assisted periodontal treatment: From bactericidal effect to local modification of the host response. Paper presented at: Sixth International Conference on Lasers in Medicine; May 7-9, 2015; Bucharest, Romania. doi:10. $1117 / 12.2189324$

23. Polansky R, Haas M, Heschl A, Wimmer G. Clinical effectiveness of photodynamic therapy in the treatment of periodontitis. J Clin Periodontol. 2009;36:575-580.
24. Dai T, Huang YY, Hamblin MR. Photodynamic therapy for localized infections - state of the art. Photodiagnosis Photodyn Ther. 2009; 6(3):170-188.

25. Tomasi C, Schander K, Dahlén G, Wennström JL. Short-term clinical and microbiologic effects of pocket debridement with an Er:YAG laser during periodontal maintenance. J Periodontol. 2006;77(1):111-118.

26. Rotundo R, Nieri M, Cairo F, et al. Lack of adjunctive benefit of Er:YAG laser in non-surgical periodontal treatment: A randomized splitmouth clinical trial. J Clin Periodontol. 2010;37:526-533.

27. Moreira AL, Novaes Jr AB, Grisi MF, et al. Antimicrobial photodynamic therapy as an adjunct to non-surgical treatment of aggressive periodontitis: A split-mouth randomized controlled trial. J Periodont. 2015;86(3):376-386.

28. Deas DE, Mealey BL. Response of chronic and aggressive periodontitis to treatment. Periodontol 2000. 2010;53(1):154-166.

29. Bizzarro S, Laine ML, Buijs MJ, et al. Microbial profiles at baseline and not the use of antibiotics determine the clinical outcome of the treatment of chronic periodontitis. Sci Rep. 2016;6:20205. doi:10.1038/ srep20205

30. Walters J, Lai PC. Should antibiotics be prescribed to treat chronic periodontitis? Dent Clin North Am. 2015;59(4):919-933. 